\title{
OPERATION OF A GYROMAGNETIC LINE WITH MAGNETIC AXIAL BIAS*
}

\author{
F.S. Yamasaki ${ }^{\xi 1}$, J.O. Rossi ${ }^{1}$, L.C. Silva ${ }^{1}$, E.G.L. Rangel ${ }^{1}$, E. Schamiloglu ${ }^{2}$ \\ ${ }^{I}$ National Institute for Space Research, Av. dos Astronautas, 1758, CEP 12227-010 \\ São José dos Campos, SP, Brazil
}

\begin{abstract}
A growing interest has been raising around the use of nonlinear transmission lines (NLTLs) for radiofrequency (RF) generation since recent results published has demonstrated great prospects for this application. The subject of this paper is about a continuous ferrite loaded nondispersive line known as a gyromagnetic nonlinear transmission line (GNLTL), biased by an axial magnetic field. This type of transmission line has demonstrated a high RF conversion efficiency (up to $20.0 \%$ ), showing a good capability of operation in a broader frequency range, between $300 \mathrm{MHz}$ and $6.0 \mathrm{GHz}$. Several authors used different approaches to study the gyromagnetic effect to understand the precession movement of the ferrite magnetic dipoles. The model proposed and studied here to analyze the GNLTL has a coaxial structure using NiZn ferrite beads distributed in a $20-\mathrm{cm}$ coaxial line at a high voltage operation. Signal results were compared to check the influence of a solenoid on the axial magnetic bias. This paper explores the oscillations generated at the output caused by the changes in the magnetic system setup. It is expected that the discussion presented here will be useful as a basis to develop a new system capable of generating RF for mobile defense platforms.
\end{abstract}

\section{I.INTRODUCTION}

The search for new technologies to reach RF generation in different ways for several applications is a constant demand in the academic community. The intention generally is to develop systems more reliable, efficient and preferable cheaper, when compared to those that already exist. Our first motivation for this project was to reach an alternative way of generating RF to establish communication between the ground station and the satellite using the GNLTL, a task made nowadays by the traveling-wave-tubes (TWTs). The GNLTL could also be used in pulsed radar satellites (Synthetic Aperture Radar - SAR), since this type of device operates in a large frequency band $(300 \mathrm{MHz}-300 \mathrm{GHz})$, generally employed in remote sensing to provide data for use in climate change, deforestation detection, development of urban areas, mobility, forecast of natural disasters and also in the military area [1]. Gyromagnetic NLTLs can produce a very broad frequency spectrum, with RF conversion efficiency of about $20 \%$ and exceeding frequencies of $6.0 \mathrm{GHz}$ [2]-[6] for potential applications in satellite communications, which require operating frequencies at least in the S-band range.

The GNLTL principle of operation has some similarities with that of the discrete NLTLs due to the sharpening of the input pulse caused by the magnetic permeability dependence on the current. When compared to the discrete lines, the gyromagnetic lines can generate stronger pulsed oscillations at higher frequencies with higher conversion efficiency as they present stronger nonlinearity of the ferrite-based inductances compared to the lines based on ceramic dielectrics [7].

In a ferrite loaded NLTL, the signal amplitude has a direct relation to the phase velocity in such a way that the voltage peak of the pulse (which propagates in the saturated ferrite) travels faster than its trough (propagates in the unsaturated ferrites), so the line nonlinearity sharpens the rise-time [8]. As the current pulse propagates down the line the magnetic permeability decreases with the current amplitude. Thus, the crest of the pulse will travel faster than its portion of lower amplitude since the propagation velocity in a nonlinear magnetic medium is given by:

\footnotetext{
* Work supported by the Southern Office of Aerospace Research and Development (SOARD - USAF) under Grants Nos. FA9550-18-1-0111 (INPE) and FA9550-15-1-0094 (UNM). Work supported by FAPESP under contract No. 2018/26086-2

छ email: fernandayamasaki@hotmail.com

${ }^{2}$ University of New Mexico, MSC 063830, Albuquerque-NM, USA
} 


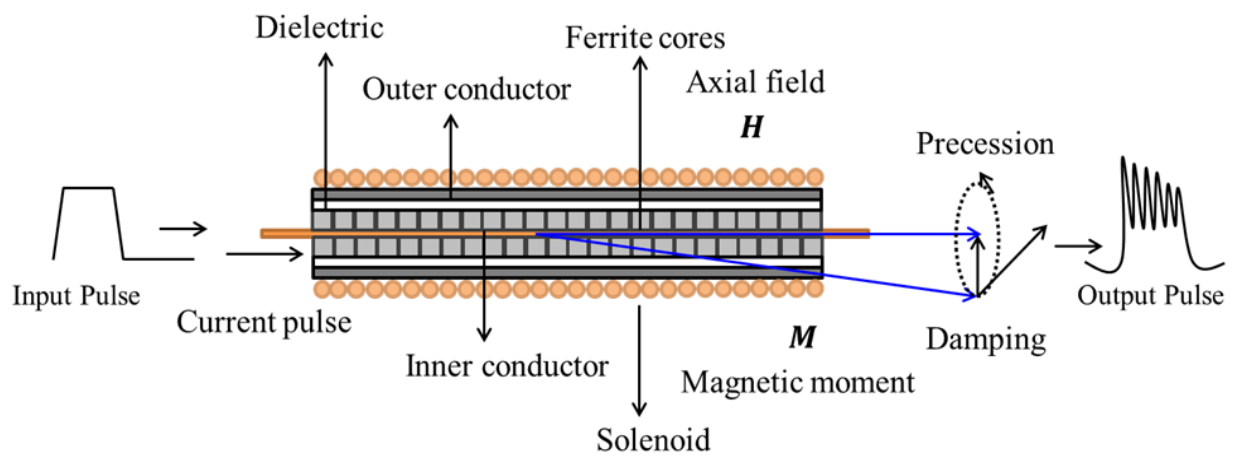

Figure 1. Precession of the magnetic moment $M$ in ferrites.

$$
v_{p}=\frac{c}{\sqrt{\varepsilon_{r} \mu_{r}(1)}},
$$

where $\mathrm{c}$ is the speed of light in vacuum, $\mu_{r}(I)$ is the relative magnetic permeability which varies with the current I and $\varepsilon_{r}$ is the relative electric permittivity.

As demonstrated in [6], the pulse rise-time reduction per meter along the line can be estimated by the difference between the propagation times of the pulse portion with lower amplitude and its peak as

$$
\Delta T=\sqrt{L C}-\sqrt{L_{\text {sat }} C},
$$

where $L$ is the line inductance per meter far from saturation, $L_{\text {sat }}$ the saturated line inductance per meter, and $\mathrm{C}$ the linear capacitance of the line per meter. The ferrite switching characteristics limit the pulse rise-time reduction due to the time it takes to switch from one state to another on the material B-H curve, which is related to the relaxation frequency of the magnetic material [8].

Also considering the propagating velocity of the signal, incident pulses with higher amplitudes generate faster output rise-times as the pulse sharpening is a result of the relation of the magnetic flux with the applied current [7],

$$
\phi(t)=L(i) i(t)
$$

\section{A. GNLTL Principle of Working}

GNLTLs generate RF or microwave oscillations based on the damped precession movement of the magnetic moments present in the ferromagnetic material, which can be used as compact, solid-state and RF source [8]. Earlier works proved that the GNLTL output frequency can be controlled by the adjustment of the bias magnetic field acting together with the high voltage amplitude of the input pulse [8], while the output power value is related to the ferromagnetic material intrinsic properties and the transmission line length [5]. Suppose that the structure shown in Fig. 1 is immersed in an axial magnetic field (generated by the solenoid around the coaxial line) $\mathrm{H}$. If a dc current pulse is injected onto the line (through the inner conductor), then the ferrite magnetic moment $\mathrm{M}$ initially aligned along the longitudinal axis, interacts with the azimuthal field that is generated around the conductor resulting in the precession of the ferrite magnetic dipoles due to the magnetic torque and with a damping factor that depends on the ferrite characteristics. This precession motion is one of the most important properties of the GNLTL because takes place along the line as soon as the current pulse starts to propagate, which results to highfrequency oscillations on the output pulse, that has a similar shape to that of the input pulse, but with a reduced rise time caused by the nonlinearity of the ferrite permeability [6].

The GNLTL frequency has a direct relation to the amplitude of the input pulse, as proved by the simplified theory developed by Romanchenko et al in [2], that calculate the oscillations of the center frequency superimposed on the amplitude of the pulse voltage propagating along the line, using as a basis for this calculation the telegraphist equation coupled to the magnetic varying flux law given by (3), as:

$$
f_{c}=\frac{\gamma}{4 \pi} \mu_{0} H_{\theta} \sqrt{1+\frac{\chi M_{s}}{\mu_{0} \sqrt{H_{\theta}^{2}+H_{z}^{2}}}}
$$

where $H_{\theta}$ is the effective magnetic field, $\gamma=1.76 \mathrm{~T} / \mathrm{rad} . \mathrm{s}$ the electron gyromagnetic ratio, $\mu_{0}=4 \pi \times 10^{-7} \mathrm{H} / \mathrm{m}$ the free space magnetic permeability, $M_{s}$ the medium saturated static magnetization (generally assumed to be $0.35 \mathrm{~T}$ for ferrites), $\chi$ is the ferrite filling factor of the NLTL. Neglecting the first term in the square root as the second one is generally much bigger and for $H_{z} \gg H_{\theta}$ obtains:

$$
f_{c} \approx \frac{\gamma}{4 \pi} \mu_{0} H_{\theta} \sqrt{\frac{\chi M_{s}}{\mu_{0} H_{z}}} .
$$

One of the parameters to be calculated to measure other line characteristics (as the impedance) is the coaxial line linear inductance per unit length:

$$
L_{0}=\frac{\mu_{0}}{2 \pi} \ln \left(\frac{b}{a}\right) \text {, }
$$

where $b$ and $a$ are the outer and inner radii of the coaxial line respectively. Another important parameter is the coaxial line linear capacitance per unit length: 


$$
C_{0}=\frac{2 \pi \varepsilon_{0}}{\ln \left(\frac{b}{a}\right)},
$$

where $\varepsilon_{0}=8.85 \times 10^{-12} \mathrm{~F} / \mathrm{m}$ is the free-space permittivity.

\section{B. GNLTL Design and Experimental Set-Up}

An experimental GNLTL has been designed and tested. The ferrite toroids used were from Feroxcube Company (see Fig.2 for size comparison), model TN9/6/3-R1 with an outer and inner diameter of $9.32 \mathrm{~mm}$ and $4.43 \mathrm{~mm}$, respectively.

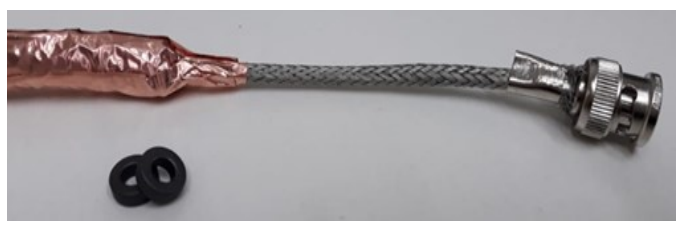

Figure 2. Ferrite beads in comparison with the size of the gyromagnetic line.

The magnetic permeability provided in the datasheet is 800. The gyromagnetic line layers were constructed using a coaxial cable, whose external insulation and braiding were peeled of $20 \mathrm{~cm}$ to place 60 ferrite beads. Two layers of Kapton were wrapped around the ferrites to isolate the beads and keep the structure rigid. Many turns of cooper tape were wound on the insulation layer of the ferrite beads to work as a braiding shield and ground. The line extremities were ended with coaxial connectors to connect the generator to the $50-\mathrm{ohm}$ attenuators in a safe mode to avoid electromagnetic interference

The test system (Fig. 3) consists of an exponential high voltage pulse generator from FID technology of $5 \mathrm{kV} / 5 \mathrm{~ns}$ to feed the gyromagnetic line and a maximum 50-A dc source to feed the solenoid for generating the axial bias magnetic field. A high voltage is needed to create a high azimuthal field inside the ferrite beads. The solenoid coil was built with 1860 turns of the copper wire AWG13 $(\phi=1.828 \mathrm{~mm})$, and an injected current around $11 \mathrm{~A}$ is needed to generate approximately $30 \mathrm{kA} / \mathrm{m}$ for H-bias. Between the ouput of of the coaxial line and the input of the 2.5-GHz digital scope from Agilent Technologies (model DSO9104A), two high voltage attenuators of the Solar Electronics Company were used to keep the input voltage to the oscilloscope below $5 \mathrm{~V}$. These $50-\Omega$ attenuators have the following specifications: $2500-\mathrm{V}$ peak, $40 \mathrm{~dB}$ of attenuation and, $2.5 \mathrm{~W}$ of average power. The inductance in $\mu \mathrm{H} / \mathrm{m}$ and the capacitance in $\mathrm{pF} / \mathrm{m}$ of the coaxial structure were calculated by using (6) and (7), respectively. Considering that the ferrite's permeability depends on the applied magnetic field, then the line impedance changes as a function of time and space when a voltage pulse with enough amplitude propagates.

The perfect impedance matching is hard to reach due to the varying line impedance during pulse application [8], but the approximately saturated value can be calculated as:

$$
Z=\sqrt{\frac{L_{0}}{c_{0}}} .
$$

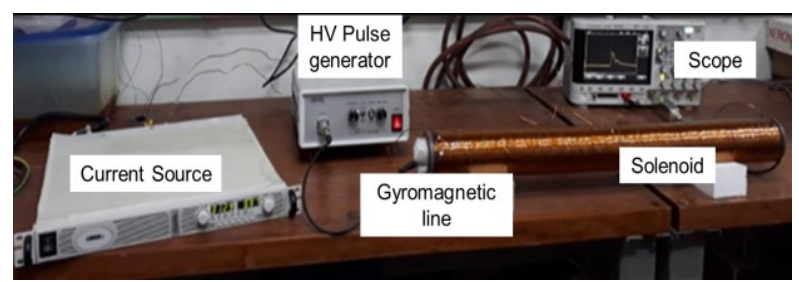

Figure 3. The test system displayed on the lab bench.

According to (6) and (7), the linear inductance and capacitance per meter for the line built are respectively $148 \mathrm{nH} / \mathrm{m}$ and $75 \mathrm{pF} / \mathrm{m}$. Thus, the line biased characteristic impedance is of the order of $44 \mathrm{ohms}$ using (8) when the magnetic permeability tends to $\mu_{0}$, if the ferrite is heavily saturated under a higher axial magnetic field.

\section{II.EXPERIMENTAL RESULTS}

In this section, an experimental analysis of the gyromagnetic line operation fed by a high voltage input pulse is given. The goal is to study the influence of the magnetic bias field on the NLTL output pulse. The first measurements given in Fig. 4 considered the signal extracted (black line) at the output with the line without magnetic bias by comparing to the output signal (blue line) with a magnetic bias of the order of $30 \mathrm{kA} / \mathrm{m}$, which corresponds to a current value of $11 \mathrm{~A}$ injected into the solenoid by the DC source.

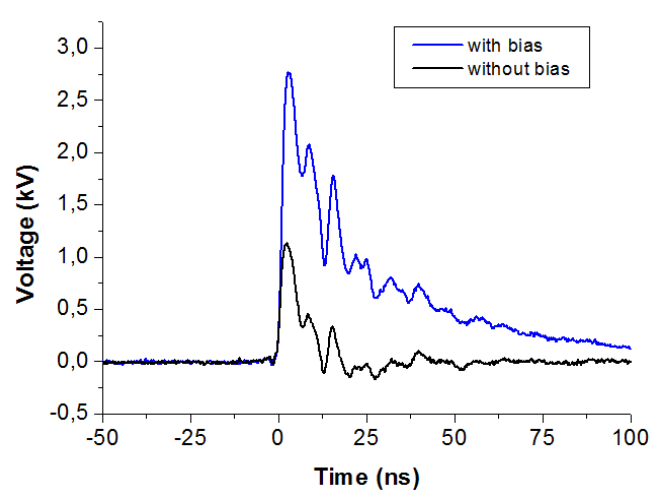

Figure 4. Comparison between both gyro output signals with and without axial bias magnetic field.

When both signals are compared, it is possible to observe that the amplitude voltage of the pulse under magnetic bias is 3 times higher than that without magnetic bias. The explanation for this is that under magnetic bias the line impedance is close to $50 \mathrm{ohms}$ and its two endings are in practice matched to the output impedance generator and the attenuator. Thus, the amplitude of the 
input generator pulse emerges on the line output with negligible drop. Besides that, one can note that more oscillations with higher voltage depth are generated in the biased case as the ferrite electron spins are not randomly oriented.

Fig. 5 shows the frequency domain (FFT) of the output pulses given in Fig. 4 with and without magnetic bias. As demonstrated by the FFT in Fig. 5, the line under magnetic bias provides a higher peak at frequency around $150 \mathrm{MHz}$ than without magnetic bias. The comparison between both signals (with and without magnetic bias) proves the influence of the axial bias on the line performance, related to the number of oscillations and voltage amplitude (see Fig. 4). Related to the FFT in Fig. 5 again, the peak in frequency is more evidenced at 150 $\mathrm{MHz}$ with the higher amplitude of this harmonic for the axially biased case.

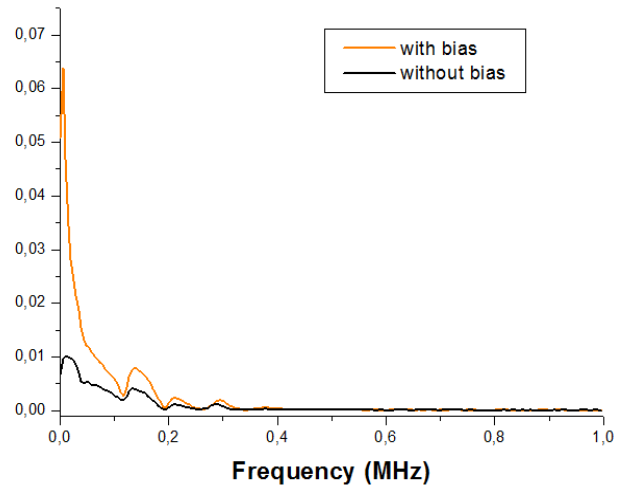

Figure 5. FFT comparison of the gyro output signals with and without axial magnetic axial bias.

As the goal of this paper was only to verify the effect of the axial bias on the line performance, the results obtained are in a good agreement with the proposal of this work. These results shown that when compared to the case without any type of bias, the gyro axially biased demonstrated an improvement on the frequency and number of oscillations with higher amplitude of harmonic generated at $150 \mathrm{~Hz}$. This is due to the fact the ferrite electron spins oriented axially by the applied external magnetic bias precess when the magnetic azimuthal field is generated by the input pulse propagation along the line, as explained previously.

\section{CONCLUSIONS}

The output frequency of the GNLTL can be controlled by the axial bias magnetic field generated by the dc source that drives the solenoid, working together with the azimuthal bias generated by the high voltage amplitude input pulse. The comparison between both signals (with and without magnetic bias) proves the great influence of the axial bias on the line performance. A high voltage for the amplitude of the input pulse is needed to generate a higher electric field inside the ferrite, which in turn will provide a high azimuthal field, at least of the order of the axial magnetic field. At high voltage operation, the frequency generated by the gyromagnetic line in our case was around $150 \mathrm{MHz}$.

\section{REFERENCES}

[1] M.M.S. Costa, O.L. Bogossian, and A. Passaro. (2015, August). Basic Requirements for synthetic aperture radar satellite mission. Presented at, Workshop on Eng. \& Space Technol. (WETE). [Online]. Available: http://urlib.net/8JMKD3MGP3W34P/3L8HBTL

[2] I.V. Romanchenko, V.V. Rostov, A.V. Gunin, and V.Y. Konev, "High power microwave beam steering based on gyromagnetic nonlinear transmission lines", J. Appl. Phys., vol.117, 214907, Jun. 2015.

[3] V.V. Rostov, N.M. Bykov, D.N. Bykov, A.V. Gunin, A.I. Klomov, O.B. Kovalchuk, V.O. Kutenkov, and I.V. Romanchenko, "Generation of sub-GW RF pulses in nonlinear transmission lines," in Proc. IEEE Int. Pulse Power Conf. (PPC), Washington, DC, Jun. 2009, pp.7073.

[4] D.V. Reale, J.M. Parson, A.A. Neuber, J.C. Dickens, and J.J. Mankowski, "Investigation of a stripline transmission line structure for gyromagnetic nonlinear transmission line high power microwaves sources". Rev. Sci. Instrum., vol. 87, 034706, Mar. 2016.

[5] D.V. Reale, J.W. Bragg, N.R. Gonsalves, J.M. Johnson, A.A. Neuber, J.C. Dickens, and J.J. Mankowski, "Bias-field controlled phasing and power combination of gyromagnetic nonlinear transmission lines". Rev. Sci. Instrum., vol. 85, 054706, May 2014.

[6] Yamasaki, F.S.; J.O. Rossi; Barroso, J. J. and Schamiloglu, E., "Operation of a Gyromagnetic Line at Low and High Voltages with Simultaneous Axial and Azimuthal Biases," in IEEE Transactions on Plasma Science, vol. 46, no. 7, pp. 2573-2581, July 2018.

[7] C.R. Spikings, N. Seddon, R.A. Ibbotson, and J.E. Dolan, "HPM Systems based on NLTL technologies, "in Proc. IET Conf. on High Power RF Technol., London, UK., 2009, pp.1-3.

[8]F.S. Yamasaki, "Simulation and experimental characterization of gyromagnetic nonlinear transmission lines for prospective aerospace applications," Ph.D. dissertation, Plasma Lab., INPE, S.J. Campos, SP, Brazil, 2017. 